\title{
O DESENVOLVIMENTO DE NOVOS SERVIÇOS EM EMPRESAS INDUSTRIAIS: ESTUDO DE CASOS EM PRODUTORES DE VEÍCULOS PESADOS
}

\author{
Eduardo Heber Gomide (e.gomide@ hotmail.com) - Universidade Federal de São Carlos \\ Glauco H. S. Mendes (glauco@ dep.ufscar.br) - Universidade Federal de São Carlos \\ Maicon Gouvea Oliveira (maicongdo@gmail.com) - Universidade Federal de Alfenas
}

\section{RESUMO}

O objetivo deste trabalho foi caracterizar e analisar o processo de Desenvolvimento de Novos Serviços no contexto de empresas industriais.

Para consecução deste objetivo, este trabalho realizou um Estudo de Caso em duas grandes empresas produtoras de veículos pesados.

O trabalho mostrou que ambas as empresas estudas tem uma ligação forte e de longa data com o processo de servitização e que este processo tornou-se uma das estratégias mais importantes no que tange à competitividade e permanência no mercado em que estão inseridas. Os estudos de caso revelaram também que as empresas, embora não tenham um processo sistematizado para o desenvolvimento de novos serviços com protocolos formatados, envolvem várias áreas no processo e têm tendência a formação de uma área responsável que reúna profissionais especializados e engajados na compreensão da demanda do mercado/cliente. $\mathrm{O}$ estudo mostrou também que existe um consenso de que o processo de desenvolvimento de novos serviços quando realizado conjuntamente ao desenvolvimento de novos produtos é vantajoso e dentre as vantagens é possível citar a diminuição de falhas na entrega, a maior personalização do pacote produto-serviço e o aumento da satisfação do cliente final.

\section{Palavras chave: Desenvolvimento de Novos Serviços, Servitização, Sistema Produto Serviços, Empresas industriais.}

Área: Inovação e a gestão do desenvolvimento de produtos e serviços em empresas no Brasil

\section{INTRODUÇÃO}

A estrutura da produção econômica mudou consideravelmente ao longo dos últimos 30 anos em todos os países europeus (NEELY, 2008) e também em países de todos os demais continentes. Em geral, a Indústria perdeu importância relativa no PIB e na geração de empregos em muitos países, enquanto o setor de serviços aumentou sua participação nesses indicadores macroeconômicos. (GALLOUJ e DEJELLAL, 2010). 
No Brasil, segundo o relatório do Ministério do Desenvolvimento, Indústria e Comércio Exterior do ano de 2016, o setor terciário passou de uma representatividade de $71 \%$ do PIB em 2014 para 72,8\% em 2015, demonstrando um pequeno aumento, mas mantendo a sua posição majoritária. (MDIC, 2014), denotando a importância dos serviços para as economias nacionais.

Serviços não são importantes apenas para as tradicionais empresas deste setor, já que as empresas industriais estão se movendo em direção à estratégia de servitização

(VANDERMERWE e RADA, 1988). Esta tendência reafirma que a indústria vem, não só aumentando a oferta de serviços adicionados aos produtos, como também dirigindo esforços para desenvolver novas ofertas de serviços, nas quais o produto em si deixa de ser o foco das relações de negócio (KINDSTROM e KOWALKOWSKI, 2014).

O pacote de produtos e serviços ofertado aos clientes para atender as suas necessidades é também chamado de Sistema Produto-Serviço, do inglês Product-Service System (PSS) (BAINES et al. 2007; BORCHARDT et al. 2010) e, neste artigo, o termo servitização será utilizado para se referir à estratégia mais geral orientada aos serviços e PSS como a oferta integrada de produto e serviço entregue ao cliente.

O processo de DNS é um fator crítico para o sucesso da entrega de uma solução integrada ao cliente (EGGERT et al. 2014), todavia, apesar da complexidade do processo, da necessidade de envolvimento com os clientes, da necessidade da simultaneidade entre a criação e a oferta (JAAKKOLA et al., 2017; MENDES et al., 2017)e apesar do aumento do número de ofertas de serviços em empresas industriais, os processos de DNS continuam sendo realizados de maneira não estruturada (GEBAUER, 2007).

Outro ponto é a divergência entre autores com relação à integração do DNS e do PDP. Aurich et al. (2004) recomenda a integração entre os processos e Gebauer et al. (2008) apontam que a integração parece ser necessária e positiva somente para alguns tipos de serviços. Gremyr et al. (2010) apontam que, embora possa haver integração entre PDP e DNS em algumas etapas do processo de desenvolvimento, a maioria das empresas industriais não possuem um processo de DNS tão estruturado como o PDP, o que reflete em diferentes níveis de maturidade para esses dois processos.

Neste contexto, ainda existem muitas dúvidas e lacunas referente a sua organização e implementação por estas empresas, sobretudo aquelas relativas à sua sistematização e sua integração com o PDP. Dessa maneira, o objetivo deste trabalho é compreender o processo de DNS em empresas industriais, bem como estas empresas organizam seus processos de Desenvolvimento de Novos Serviços e como este processo é integrado ao processo de Desenvolvimento de Novos Produtos.

\section{REVISÃO DE LITERATURA}

\subsection{Servitização e PSS: benefícios e desafios}

O termo Servitização foi usado pela primeira vez por Vandermerwe e Rada (1988), que o definem como a oferta de um pacote integrado de produtos, serviços, conhecimento e suporte ao cliente a fim de agregar valor ao negócio principal da empresa. Para Baines et al. (2007), um tipo especial de servitização é o Sistema Produto Serviço, do inglês ProductService System (PSS). Mais recentemente, Kowalkowski et al. (2017) aponta que servitização é reconhecida como o processo transformacional no qual uma empresa industrial altera seu 
modelo de negócio centrado no produto para um modelo de negócio centrado em serviços. Portanto, empresas servitizadas movem-se para a oferta de sistemas produtos serviços (PSS).

Para Morelli (2003), a servitização (servitization) consiste na evolução de uma oferta material para uma posição em que o componente de material é inseparável dos serviços. Similarmente, produtização (productization) refere-se à inclusão de produtos a uma base de serviços. Essas duas estratégias caminham na concepção de um novo modelo de negócio, pautado na oferta única e integrada de produtos e serviços (BAINES et al., 2007).

O termo PSS teve sua origem nos anos 1990, no norte da Europa, associado à ecologia industrial e centrado no conceito de vender desempenho por meio de uma orientação para serviços em vez de vender apenas bens (SPRING e ARAÚJO, 2009). Contudo, outros termos também estão associados ao conceito de PSS, tais como produtos funcionais (ALONSORASGADO e THOMPSON, 2006) e vendas funcionais (LINDAHL e ÖLUNDH, 2001; LINDAHL et al., 2006). Em negócios entre empresas industriais (business-to-business), muitos autores adotam o termo PSS industrial (MEIER et al., 2010; MÜLLER e STARK, 2010; CEDERGREN et al., 2012).

\subsection{A estratégia de DNS em empresas industriais}

A inovação em empresas industriais é tradicionalmente entendida pela pesquisa e desenvolvimento (P\&D) com o intuito de criar e comercializar produtos com novas tecnologias (GEBAUER et al., 2012). Logo, muitas empresas industriais estão desenvolvendo serviços para seus clientes.

Para acompanhar esta tendência, as empresas industriais precisam criar e desenvolver estratégias de serviços que viabilizem esta transição. Para tanto, dentre outros, os trabalhos de Baines e Lightfoot (2013), Fischer et al. (2014) e Smith et al. (2014) auxiliam na definição de estratégias de serviços voltadas para este tipo de empresa.

O trabalho de Baines e Lightfoot (2013) também auxilia nas estratégias de serviços que podem ser adotadas por empresas industriais. Tais empresas podem se tornar provedores de serviços básicos, provedores de serviços intermediários e ou provedores de serviços avançados. Os Serviços Básicos referem-se aos serviços que são adicionados e são diretamente associados aos produtos. Nesta categoria estão, por exemplo, os serviços oferecidos relacionados ao fornecimento dos produtos e de peças de reposição para o cliente. Os Serviços Intermediários referem-se aos serviços mais ou menos complexos que são atrelados aos produtos. Neste caso, o foco é para o uso correto dos produtos e sua manutenção e disponibilidade. Incluem-se os serviços básicos, mas também serviços de manutenção corretiva e preventiva, serviços de suporte ao cliente, treinamento de clientes etc. Nos Serviços Avançado, a ênfase se afasta do foco no produto e se concentra mais em consequiência de seu desempenho. No geral, o provedor do serviço entende perfeitamente a natureza do negócio do cliente e os processos que envolvem a oferta do serviço e, consequentemente, pode oferecer "uma competência" ao cliente, por exemplo.

Mais recentemente, os trabalhos de Fischer et al. (2014) e Smith et al. (2014) apresentam um conjunto de estratégias de serviços que são baseadas na evolução gradual da oferta de serviços atrelados aos produtos. As etapas compreendidas são: asset sales, asset recovery, asset availability, outsourcing partner e development partner. A Asset sales (venda de ativos) que refere-se à proposta de valor pela qual uma empresa industrial concentra-se basicamente na venda do produto e de serviços básicos de pós-venda. A Asset recovery (recuperação de ativos) que tem base na oferta dos produtos e adotam a estratégia de oferecer 
serviços pós-venda para garantir a manutenção e recuperação dos produtos. A Asset avaialability (disponibilidade de ativos) que trabalha com serviços pós-venda e fornece o know-how para que uma empresa industrial aumente a abrangência e a complexidade dos serviços prestados aos seus clientes. Os Outsourcing partner (parceiros de terceirização) que se referem à proposta de valor baseada na terceirização e atendem a necessidades dos clientes como reduções de capital empregado ou investimentos em ativos fixos. Neste caso, o cliente transfere a responsabilidade de parte do processo para a empresa provedora do produto. Os Development partner (parceiros de desenvolvimento) que se referem à proposta de valor de oferecer capacidades de engenharia e P\&D como um serviço aos clientes. Neste caso, a empresa industrial assume (como um serviço prestado), atividades de desenvolvimento de produtos e tecnologia para seus clientes.

\section{MÉTODO}

Foram realizados dois estudos de caso em dois fabricantes de veículos pesados brasileiros, ambos com atuação global e presença consolidada no mercado brasileiro. Por solicitação das empresas (entrevistados), os reais nomes das empresas foram omitidos e estas foram chamadas de "Empresa A" e "Empresa B".

A Empresa A é um dos maiores fabricantes mundiais de caminhões, ônibus, equipamentos de construção, motores marítimos e industriais. Com sede em Gotemburgo na Suécia, emprega cerca de 95.000 pessoas ao redor do mundo, tem fábricas em 18 países e atua em 190 mercados. No Brasil, a empresa foi constituída no final dos anos 70 e desde então a marca inova introduzindo veículos e serviços que trazem novos conceitos ao mercado de transporte de carga do país e foi líder no mercado de caminhões pesados em 2016. A Empresa A tem sua sede no Brasil situada em Curitiba - PR, onde produz caminhões, ônibus e motores. Há unidades também em Pederneiras - SP (produção de equipamentos de construção) e São José dos Pinhais - PR (centro de distribuição de peças). Ao todo, a empresa conta com cerca de 5.000 funcionários diretos no país.

A Empresa B é um dos principais grupos de engenharia mecânica e de veículos comerciais da Europa com mais de 250 anos de história e mais de 55.000 funcionários espalhados por 180 países, globalmente as atividades do grupo estão espalhadas em duas áreas de negócios: Veículos Comerciais (Caminhões \& ônibus) e Power Engineering (Diesel \& Turbo Renk). Na América Latina, o subgrupo que é a unidade de análise deste estudo, é formado após a aquisição das atividades de caminhões e ônibus brasileiros da Volkswaggen e concentra esforços no fornecimento de caminhões de 5 a 90 toneladas e ônibus e serviços de leasing operacional e contratos de manutenção. No Brasil a Empresa B conta com uma planta de produção em Resende - RJ e sede administrativa em São Paulo - SP, ao todo conta com 52 colaboradores contratados e mais essa mesma quantia em prestadores de serviços e terceirizados, além de uma ampla rede de concessionários espalhados pelo país.

Para a Empresa A, três colaboradores foram selecionados, sendo o Gerente-geral de pós-vendas e 2 Coordenadores Técnicos. Na Empresa B foram entrevistados dois colaboradores, sendo um Gerente-Geral do Departamento de Pós-vendas e um Coordenador Técnico do Subdepartamento de Marketing.

As entrevistas tiveram duração média de 60 minutos e o questionário semiestruturado foi formatado de maneira a caracterizar o PSS da empresa e o Desenvolvimento de Novos Serviços. Os dados coletados durante as entrevistas foram analisados antes da exposição no próximo capítulo por meio do método proposto por Bardin (2008). 


\section{DISCUSSÃO DOS DADOS}

\subsection{Identificação e Caracterização da Área Responsável pelo DNS}

Na Empresa A o desenvolvimento de novos serviços fica sob responsabilidade do setor de Pós-vendas, que também cuida dos projetos de leasing operacional e dos planos de manutenção programada e conta com o envolvimento de outras 2 áreas, vendas e a locação. A área de pós-vendas gerencia o andamento dos projetos, a área de vendas por estar diretamente ligada com os clientes, tem papel importante em alimentar o projeto com a opinião e as percepções dos clientes, e a área de locação contribui com as demandas específicas de clientes que buscam pacotes de produtos e serviços, principalmente. O setor possui ramificações em algumas filiais com gerentes responsáveis pelo pós-vendas/DNS (peças e serviços) e seguindo a hierarquia temos alguns coordenadores de oficina e de peças que também estão envolvidos no processo.

Para a Empresa B, assim como na Empresa A, estes projetos ficam sob a responsabilidade de um setor denominado de pós-vendas, esse setor é subdividido e tem a responsabilidade também sobre o marketing, as vendas e o próprio pós-vendas, onde existem colaboradores com maior e menor grau de envolvimento com o DNS. Este setor fica alocado em São Paulo e conta com apoio das equipes que formam a sua rede de concessionários. As equipes de concessionários são responsáveis por alimentar os projetos com os feedbacks dos clientes, apoiar os estudos de viabilidade econômica dos projetos e, sobretudo entender e acompanhar as operações dos clientes.

\subsection{Caracterização do projeto do novo serviço}

Embora se observe uma tendência para formação de uma área específica que reúna especialistas em DNS em um setor específico, em ambas as empresas, ainda não existe esta mesma tendência para um protocolo estruturado para este processo, fato surpreendente para empresas consideradas grandes, seja em número de clientes ou em potencial de vendas.

Para ambas as empresas, o que pode ser observado é uma ligeira preocupação maior com as fases iniciais do processo de DNS, influenciadas e dependentes basicamente do contato com os clientes, que pode ser explicada pela preocupação da empresa em gerir melhor os gastos com o desenvolvimento e melhorando a rentabilidade dos projetos.

O projeto de DNS para a Empresa A parte da geração de ideias de novos serviços que é baseada no relacionamento entre a empresa e o cliente, sem uma técnica específica. Um ponto interessante da fase de geração de ideias, são os encontros realizados, pelo menos 3 vezes ao ano, com todos os gerentes de pós-vendas para debater todas as demandas advindas dos clientes e selecionar quais seguirão para tornarem-se projetos de DNS e posteriormente projetos-piloto nas filiais.

A fase posterior é seleção das ideias, onde a Empresa A faz uso de uma ferramenta online, semelhante a um fórum de debate, onde as áreas envolvidas debatem e decidem quais terão prioridades de seguir adiante. A duração dos processos de desenvolvimento de novos serviços, partindo da geração de ideias até a implantação de projetos-pilotos é, em média, de aproximadamente 8 a 12 meses.

Igualmente para a Empresa B, não existe um processo estruturado para o DNS e a relação empresa x cliente é muito importante como maneira de garantir a geração de ideias para o DNS. A elaboração do projeto é justificada com uma série de informações que são 
extraídas do cliente, informações estas que são relevantes basicamente para o dimensionamento (estratégico e financeiro) do projeto e a maneira como essas informações são extraídas não é por meio de uma ferramenta específica e depende, basicamente, da habilidade do responsável pelo projeto. Uma peculiaridade da empresa B para a geração de ideias é o que ela chama de "clínica de clientes", um grupo focal com clientes utilizando a técnicas do QFD (Quality Function Deployment) para identificar as necessidades dos clientes e do mercado.

O tempo médio de duração do processo todo para a Empresa B é maior, podendo levar de 6 meses a 5 anos para serem finalizados e o critério para a escolha de prioridades dos projetos é baseado no timing estratégico da empresa.

Outro ponto comum é a documentação do processo que é realizada por meio do registro de um Business Case detalhado com o memorial dos estudos realizados de maneira que justifique todo esforço e investimento no projeto de DNS.

Os projetos-pilotos são usados para testar, de maneira prática um produto ou um serviço antes dele ser lançado em grande escala para todos os clientes, dessa maneira, a Empresa A e a B realizam testes-piloto durante o desenvolvimento de novos serviços e que estes testes tem duração variável dependendo do grau de complexidade do serviço. Um peculiaridade dos testes-piloto realizados pela Empresa B é a realização em duas fases com clientes parceiros, a primeira com protótipos iniciais e depois com o protótipos com maior nível de maturidade.

A fase seguinte refere-se à avaliação da entrega e a verificação de possíveis melhorias, a Empresa A realiza uma pesquisa anual com todos os seus clientes avaliando a opinião sobre os seus serviços e também avalia a opinião dos clientes com relação às outras marcas concorrentes. A Empresa B faz essa verificação avaliando constantemente o nível de satisfação dos clientes com relação às soluções implementadas e para isso tem uma área que faz esse monitoramento constante e que recebe o feedback dos clientes, além disso a empresa conta com uma equipe de consultores nos escritórios regionais que realiza visitas frequentes aos clientes com o objetivo de estar próximo dos clientes, identificar possíveis problemas e possíveis oportunidades de melhoria.

\subsection{Integração dos processos de DNS e PNP}

Para as duas empresas, a integração do PDP e do DNS está presente em alguns serviços e é verificada, principalmente, durante as etapas iniciais do processo. Para as empresas, embora o PDP e DNS estejam sob a responsabilidade de áreas distintas, algumas outras áreas que são comuns aos dois processos participam direta ou indiretamente em alguns momentos.

Existe o consenso entre as duas empresas de que alguns produtos e serviços são desenvolvidos especificamente para trabalharem em conjunto e por isso requerem maior integração durante a fase de desenvolvimento, e outros são desenvolvidos por uma demanda posterior ao lançamento do produto no mercado e isso justifica a pouca ou a inexistência de integração.

As áreas que comumente se integram durante o processo é a área de pós-vendas, tida geralmente como a responsável pelos projetos de DNS, com a área financeira, de vendas e de qualidade. 
Como vantagens à integração dos dois processo (PDP e DNS), a Empresa A coloca do que esta atitude é mais eficiente em atender às expectativas dos clientes e que isto é um fator que pode influenciar os clientes na escolha por uma empresa a outra, já a Empresa B enfatiza as vantagens a cerca da questão financeira, que coloca que a integração possibilita que a empresa possa identificar todas as necessidades e oportunidades de melhoria e mudanças em tempo hábil e que dessa maneira o projeto possa ser alterado sem um alto impacto no seu custo.

A cerca das desvantagens, apenas a Empresa B cita que a integração dos processos pode gerar um custo de investimento mais alto e isto poderia se tornar um empecilho para a aprovação por parte do setor financeiro, no entanto considera importante e inerente ao processo que isso ocorra.

\section{CONCLUSÕES}

Pode-se concluir que a servitização dos negócios, sobretudo das indústrias, reflete em um grande desafio, haja visto o requerimento de mudanças em vários aspectos dos seus tradicionais negócios de venda de produtos.

Para a consecução dos objetivos a que este trabalho se propôs, um estudo de caso múltiplo em duas empresas produtoras de veículos pesados sob servitização foi realizado. Os dados coletados foram apresentados de maneira a elucidar a realidade dessas empresas através das características dos modelos de negócio e dos processos que utilizam, bem como demonstrar as motivações e os desafios que elas enfrentam.

As empresas estudadas, embora de grande porte, não possuem uma área estruturada e dedicada apenas para o DNS, assim como, não se evidenciou o uso de protocolos estruturados com etapas bem definidas.

Em ambas as empresas observam-se que a área de pós-vendas é escolhida como sendo a responsável pelo DNS pelo fato de seu maior contato com os clientes. Esse estreitamento da ligação empresa x cliente é fundamental para o processo por intensificar a geração de insights, facilitar a empresa a traduzir as demandas do mercado e também de aferir a satisfação dos clientes com os serviços e produtos disponibilizados.

Vale-se dizer que o conhecimento da equipe que integra o processo é fundamental para o sucesso dos projetos de DNS haja visto que em vários pontos as decisões e os passos seguintes são definidos com base na experiência dos responsáveis pelo processo.

Por fim e não menos importante, o consenso positivo sobre a integração dos processos de PDP e DNS apresentado pelas vem corroborar com as literaturas mais recentes colocando que o desenvolvimento conjunto é responsável pela diminuição de falhas na entrega final pro cliente, pela maior personalização do pacote de serviços e pelo aumento da satisfação do cliente final.

Sobretudo, este trabalho tem limitações que devem ser consideradas. A primeira limitação diz respeito ao número de entrevistados que, apesar de relevantes, este número não oferece embasamento suficiente para que as conclusões deste trabalho sejam generalizáveis a outras empresas e indústrias. Outro ponto é com relação ao entendimento de como as outras áreas da empresa interferem no processo de DNS. Dessa maneira, seria interessante, por exemplo, entender a questão dos fornecedores externos, $\mathrm{RH}$, e Compras participam da estratégia de servitização e qual o impacto da estratégia nessas áreas. 
Com base nessas limitações, a sugestão é que em uma pesquisa futura seja realizada com um Estudo de Casos Múltiplos que tenha acesso a colaboradores de todas as Áreas que interferem no DNS (Compras, RH e Fornecedores e Externos) e que consiga, de fato, oferecer uma visão que ampla e generalizável deste processo.

\section{REFERÊNCIAS BIBLIOGRÁFICAS}

ALONSO, M. P. Product service system: benefits and barriers. (MSc Thesis. 105p). School of Applied Sciences. Cranfield University. London, UK. 2007.

ALONSO-RASGADO, T., THOMPSON, G. A rapid design process for Total Care Production creation. Journal of Engineering Design, 17, 6, pp. 509-531. 2006.

AURICH, J.C.; FUCHS, C.; DEVRIES, M.F. An Approach to Life Cycle Oriented Technical Service Design. CIRP Annals - Manufacturing Technology, v. 53, n.1, p.

151-154. 2004.

BAINES, T.; LIGHTFOOT, H. Made to Serve: How Manufacturers Can Compete Through Servitization and Product - Service Systems, John Wiley \& Sons, Inc., Hoboken, NJ, USA. 2013.

BAINES, T. S.; LIGHTFOOT, H. W.; EVANS, S. State-of-the-art in product-service systems. Proceedings of the Institution of Mechanical Engineers, Part B: Journal of Engineering Manufacture, v. 221, n. 10, p. 1543-1552, 2007.

BARDIN, L. Análise de Conteúdo. Lisboa, Portugal; Edições 70, LDA, 2008.

BEUREN, F. H., FERREIRA, M. G. G.; MIGUEL, P. A. C. Product-service systems: a literature review on integrated products and services. Journal of Cleaner Production, v.47, p. 222-231. 2013.

BORCHARDT, M.B.; SELLITTO, M.A.; PEREIRA, G.M. Sistemas produto-serviço: referencial teórico e direções para futuras pesquisas. Revista Produção on-line, v.10 n.4, p837-860, 2010.

CEDERGREN, S. I.; ELFVING, S. W.; ERIKSSON, J.; PARIDA, V. Analysis of the industrial product-service systems (IPS2) literature: a Systematic Review. Proceedings International Conference on Management of Innovation and Technology (ICMIT) 2012, IEEE, p. 733-740, [S.l: s.n.]. 2012.

EGGERT, A; HOGREVE, J; ULAGA, W; MUENKHOFF, E. Revenue and profit implications of industrial service strategies, Journal of Service Research, Vol. 17, No. 1, pp. 23-39. 2014.

FISCHER, T.; GEBAUER, H.; FLEISCH, E. Service Business Development Strategies for Value Creation in Manufacturing Firms. Cambridge University Press. 2014.

GALlOUJ F.; DJELlaL F. The Handbook of Innovation and Services: a multidisciplinary perspective, Edward Elgar Publishers. 2010.

GEBAUER, H. Identifying service strategies in product manufacturing companies by exploring environment-strategy configurations. Industrial marketing management. V. 37,p. 278-291. 2008. 
GEBAUER, H. An investigation of antecedents for the development of customer support services in manufacturing firms. Journal of Business-to-Business Marketing, Vol. 14 No. 3, pp. 59-96. 2007.

GEBAUER, H.; PAIOLA, M.; EDVARDSSON, B. A capability perspective on service business development in small and medium-sized suppliers. Scandinavian Journal of Management, Vol. 28, No. 4, pp. 321-339. 2012.

GEBAUER, H.; BRAVO-SANCHEZ, C.; FLEISCH, E. Service strategies in product manufacturing companies. Business Strategy Series, V. 9, N. 1, p. 12-20 2008.

GEBAUER, H; KREMPL, R; FLEISCH, E. Service development in traditional product manufacturing companies. Eur. J. Innov. Manage., 11(2): 219-240. 2008.

GREMYR, I; LÖFBERG, N.; WITELL, L. Service innovations in manufacturing firms. Managing Service Quality, Vol. 20 No. 2, pp. 161-175. 2010.

JAAKKOLA， E.; MEIREN， T.; WITELL， L.; EDVARDSSON， B.; SCHÄFER， A.; REYNOSO, J.; SEBASTIANI, R.; WEITLANER, D. Does one size fit all? New service development across different types of services. Journal of Service Management, v. 28, n. 2, p. 329-347, 2017.

JAMES, P.; HOPKINSON, P. Service Innovation for Sustainability: a new option for UK Environmental Policy? Bradford University, Bradford. 2002.

KINDSTRÖM, D; KOWALKOWSKI, C. Service innovation in product-centric firms: a multidimensional business model perspective. Journal of Business \& Industrial Marketing, Vol. 29 Iss 2 pp. $96-111.2014$.

KOWALKOWSKI, C.; GEBAUER, H.; KAMP, B.; PARRY, G. Servitization and deservitization: Overview, concepts, and definitions. Industrial Marketing Management, v.60. p. 4-10. 2017.

LINDAHL, M.; ÖLUNDH, G. The meaning of functional sales, Life Cycle Engineering. Challenges and Opportunities. In: Proceedings of the 8th International Seminar on Life Cycle Engineering. CIRP. Anais... [S.l: s.n.]. , 2001.

LINDAHL, M.; SUNDIN, E.; SHIMOMURA, Y.; SAKAO, T. An interactive design model for service engineering of functional sales offers. In: International Design Conference Design 2006, Anais... [S.1: s.n.]., 2006.

MANZINI, E.; VEZZOLI, C.; CLARK, G. Product service systems: using an existing concept as a new approach to sustainability. Journal of Design Research. v. 1, n.2. 2001.

MDIC - MINISTÉRIO DO DESENVOLVIMENTO, INDÚSTRIA E COMÉRCIO EXTERIOR. Secretaria de Comércio Exterior. Departamento de Defesa Comercial - Brasília: MDIC, 2014- $71 \mathrm{p}$.

MEIER, H.; ROY, R; SELIGER, G. Industrial Product-Service Systems - IPS2. CIRP Annals Manufacturing Technology, v. 59, n. 2, p. 607-627, 2010.

MENDES, Glauco HS et al. Uncovering the structures and maturity of the new service development research field through a bibliometric study (1984-2014). Journal of Service Management, v. 28, n. 1, p. 182-223, 2017.

MONT, O. Clarifying the concept of product-service system. Journal of Cleaner Production. v.10, p. 237-245, 2002. 
MÜLLER, P.; STARK, R. A generic PSS development process model based on theory and an empirical study. In: International Design Conference Design 2010 Anais... [S.l: s.n.]. , 2010.

MORELLI, N. Product-service systems: a perspective shift for designers. A case study: the design of a telecentre. Design Studies. v.24, p.73-99, 2003.

NEELY, A. The servitization of Manufacturing: An Analysis of Global Trends. In: The $14^{\text {th }}$ European Operation Management Association Conference. 2008. Anais... [S.1: s.n.]., 2008.

SAKAO, T.; SHIMOMURA, Y. Service Engineering: a Novel Engineering Discipline for Producers to Increase Value Combining Service and Product, Journal of Cleaner Production, v;15, p. 590-604. 2007.

SCHUH, G; KLOTZBACH, C.; GAUS, F. Service Provision as a Sub-model of Modern Business Models. Production Engineering, v. 2, n 1, p. 79-84, 2008.

SMITH, L.; MAULL, R. Servitization and operations management: a service dominantlogic approach. International Journal of Operations \& Production Management, Vol. 34, No. 2, p. 242-269. 2014.

SPRING, M.; ARAÚJO, L. Service and products: rethinking operations strategy. Journal of Operations \& Production Management, v. 29, n. 5, p. 444-467. 2009.

SRIVASTAVA, R.K.; TASADDUQ A. S.; FAHEY, L. Market-Based Assets and Shareholder Value: A Framework for Analysis. Journal of Marketing, v.62 (01), p. 2-18. 1998.

TUKKER, A. Product services for a resource-efficient and circular economy - A review. Journal of Cleaner Production, V.97, p.76-91. 2015.

TUKKER, A; TISCHNER, U. Product-services as a research field: past, present and future. Reflections from a decade of research. Journal of Cleaner Production, v. 14, n.17, p. 1552-1556, 2006.

UNEP - United Nations Environment Programme. Product-Service Systems and Sustainability: Opportunities for Sustainable Solutions. INDACO Department, Politecnico di Milano, Milão: 2002.

VANDERMERWE, S., RADA, J. Adding value by adding services. European Management Journal. v. 6, p. 314-323, 1988. 